\title{
Evolution of the Seminal O'Hara Rudy Model to More Accurately Simulate the Electrophysiology of Human Ventricular Cardiomyocytes
}

\author{
Chiara Bartolucci ${ }^{1}$, Michelangelo Paci ${ }^{2}$, Jari Hyttinen ${ }^{2}$, Elisa Passini ${ }^{3}$, Stefano Severi ${ }^{1}$ \\ ${ }^{1}$ University of Bologna, Cesena, Italy \\ ${ }^{2}$ Tampere University, Tampere, Finland \\ ${ }^{3}$ University of Oxford, Oxford, United Kingdom
}

\begin{abstract}
The aim of our work consists in developing a new model of the human ventricular cardiomyocyte, based on the O'Hara-Rudy model (ORd), to improve, in particular, the action potential duration (APD) dependence on the extracellular $\mathrm{Ca}^{2+}$ concentration $\left(\mathrm{Ca}_{o}\right)$. Moreover, the new model reproduces all the available experimental data on APD rate dependence, APD restitution, drug block effects, etc., when the experimental extracellular ionic concentrations are carefully reproduced in simulations.

The main differences between our and the ORd model are: a novel Markovian formulation for the L-type $\mathrm{Ca}^{2+}$ current; the Markovian rapid delayed rectifier $\mathrm{K}^{+}$current formulation published by Li et al.; a new formulation of the $\mathrm{Ca}^{2+}$ release from the sarcoplasmic reticulum. Several model parameters were modified.

Our BS (Bartolucci-Severi) model successfully improved the ORd, one of the most detailed, used and influent models in computational cardiology, by reproducing the $A P D-C a_{o}$ relationship while keeping all the original model features tested in the appropriate experimentally-matched conditions. Furthermore, the BS was suitability as baseline for the generation of in silico populations of models and for reproducing cardiac abnormalities such as early afterdepolarizations.
\end{abstract}

\section{Introduction}

In the last century, AP mathematical models have gained a relevant role in the investigation of cellular electrophysiology. Their possible application to the heart has been soon realized and right from the beginning, cardiac cell modelling allowed to gain insights by predicting phenomena which have been later confirmed experimentally.

Due to the limited availability of human cardiomyocytes for experimental research, most electrophysiological models had been formulated for animals (mouse, guinea pig, rabbit, dog, etc.). However, animal and human cardiomyocytes differ in major aspects, such as action potential shape and duration, range of normal heart rates, action potential restitution and relative importance of ionic currents in the action potential generation. As all these factors may influence the mechanism of arrhythmias initiation and dynamics, simulation results obtained with animal models may prove inadequate to represent phenomena observed in humans.

In recent years, more and more data on human cardiac ionic currents have been gathered from human cardiomyocytes and several new models have been developed to describe the origins of the human cardiac action potential, an important step towards a wider application in clinical practice.

Currently, the "gold standard" for in silico human ventricular cellular electrophysiology is the O'Hara-Rudy model (ORd) [1] which was developed and validated by using an extensive dataset, including many previously unpublished experimental data, from more than 100 undiseased human hearts. However, since mathematical models will always be approximate representations of the "real systems", it is necessary to constantly develop and improve them, depending on the specific question to address.

Focusing on some mechanisms that are not well reproduced by the ORd model, such as the dependence between APD and $\left[\mathrm{Ca}^{2+}\right]_{\text {o }}$ variations, we present an evolution of the model (BS model) that is able to correct this aspect while not altering the model behaviour in control conditions, as similarly done for older models in previous works $[2,3]$.

Moreover, we use the new model to construct a population of models $[4,5]$ which is used to evaluate the ability to reproduce cardiac abnormalities, such as early afterdepolarizations (EADs).

\section{Methods}

The ORd human ventricular AP [1] model was chosen as basis for this study because of the large number of 
human ventricular experimental data obtained from more than 140 hearts used in its construction and evaluation, and its choice within the CiPA initiative [6].

However, most of the published human AP models, including $\mathrm{ORd}$, have not taken into account the dependence between APD and $\left[\mathrm{Ca}^{2+}\right]_{0}$ variations, and therefore respond in a non-physiological way, i.e. increases in $\left[\mathrm{Ca}^{2+}\right]_{\mathrm{o}}$ lengthen APD, and viceversa.

The process to improve the ORd model has involved the reformulation of the L-Type $\mathrm{Ca}^{2+}$ current, which has been inherited from [8,9], where a Markov model description was used.

In order to maintain a physiological calcium handling, the parameters involved in the process have been modified. In particular:

- $\quad$ the SR was reduced to a single compartment (with a consequent rescaling in the cell geometry)

- the SR calcium release equations were replaced with a description similar to the one presented in Paci et al. [10]

- $\quad$ the rates in the CDI $\left(\mathrm{Ca}^{2+}\right.$-dependent inactivation) loop versus VDI (voltage-dependent inactivation) loop are less faster than in [8]

- the background $\mathrm{Ca}^{2+}$ maximal current was increased

- $\mathrm{Ca}^{2+}$ diffusion from the sub-sarcolemma space to the cytosol was speeded up

- $\quad$ the SERCA pump maximal current was increased

- the $\mathrm{Na}^{+} / \mathrm{Ca}^{2+}$ exchanger maximal current was increased

- $\quad$ the L-type $\mathrm{Ca}^{2+}$ channel permeability was reduced.

Recently Li et al. [11] published a modification to the ORd model consisting in the replacement of the $\mathrm{I}_{\mathrm{Kr}}$ with a Markov formulation; we decided to take this improvement also in the present model. To gain a good fitting of the steady state APD rate dependency and the S1S2 APD restitution experimental data, some others changes were addressed:

- $\quad$ increase of late sodium maximal current

- $\quad$ increase of $\mathrm{I}_{\mathrm{Kr}}$ maximal current

- $\quad$ increase of $\mathrm{Na}^{+} / \mathrm{K}^{+}$ATPase maximal current;

- reduction of inward rectifier potassium maximal current and increase of its instantaneous rectification slope.

Since the APD rate dependence and APD restitution experimental data, which have been used for validating the

model, were obtained with $\left[\mathrm{K}^{+}\right]_{\mathrm{o}}=4 \mathrm{mM}$ [1], we modified the $\left[\mathrm{K}^{+}\right]_{\mathrm{o}}$ concentration accordingly and a shift of the membrane potential dependence of the involved currents $\left(\mathrm{I}_{\mathrm{to}}, \mathrm{I}_{\mathrm{K} 1}, \mathrm{I}_{\mathrm{Ks}}\right)$ was introduced to compensate the liquid junction potential, which was not considered by O'Hara et al. when reporting experimental data (see [1], Comments section).

Finally, we used the BS model to construct a population of non-diseased AP models accounting for biological variability. Eleven conductances were sampled from a range of $[0.2 ; 2]$ using the Latin Hypercube Sampling [12]. The calibration was based on the AP biomarkers from human adult ventricular cardiomyocytes [1] (summarized in [13]). We also used the population of models approach to evaluate the occurrence of cardiac abnormalities, such as EADs and repolarization failures (RFs). The simulated protocol aimed to reproduce the administration of $0.1 \mu \mathrm{M}$ dofetilide at a cycle length of $4000 \mathrm{~ms}$; the $\mathrm{I}_{\mathrm{Kr}}$ drug binding values reported by Dutta et al. [14] and the same extracellular concentration experimentally used by Guo et al. [15] were used.

Model differential equations were implemented in Matlab (Mathworks Inc., Natick, MA, USA) and solved with a variable order solver (ode15s), based on numerical differentiation formulas [16]. Simulations were run with the original and modified models at variable $\left[\mathrm{Ca}^{2+}\right]_{\mathrm{o}}$ in the range $0.9-2.4 \mathrm{mM}$. Pacing was maintained until steady state AP was reached (1000 s) and APDX was measured once membrane voltage reached $\mathrm{X} \%$ of the resting value (as in [1]).

\section{Results}

\subsection{APD vs $\left[\mathrm{Ca}^{2+}\right]_{\mathrm{o}}$}
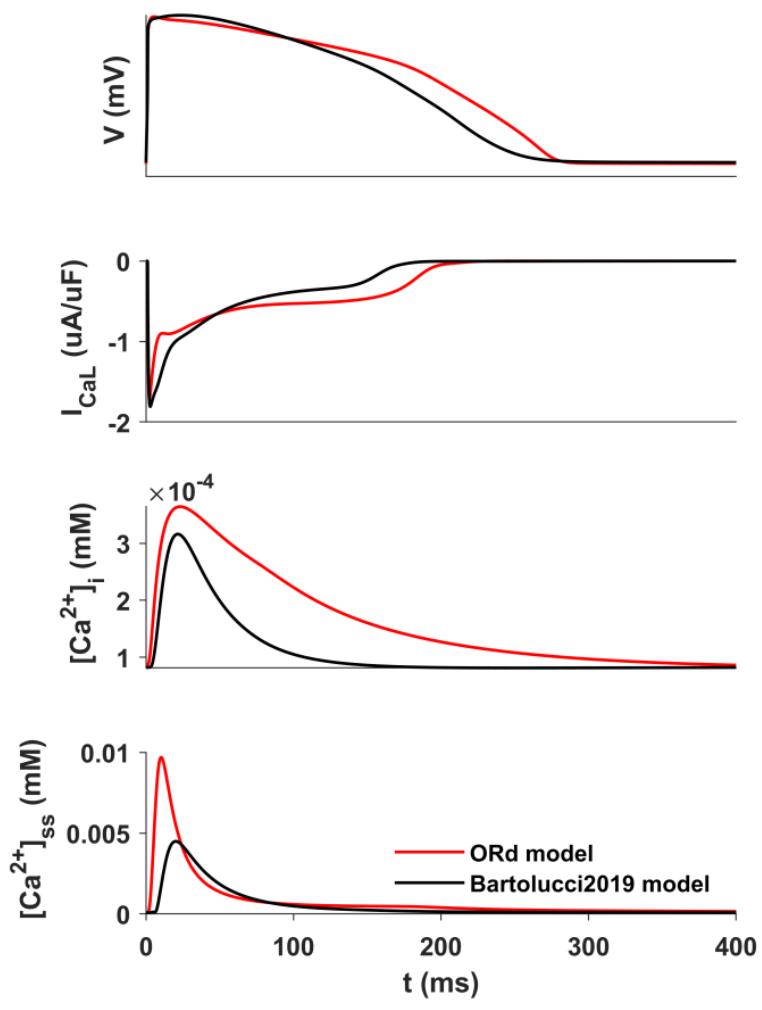

Figure 1. Comparison of the AP, $\mathrm{I}_{\mathrm{CaL}},\left[\mathrm{Ca}^{2+}\right]_{\mathrm{i}},\left[\mathrm{Ca}^{2+}\right]_{\mathrm{ss}}$ for ORd and $\mathrm{BS}$ models $(\mathrm{CL}=1 \mathrm{~s}$ in steady state). 


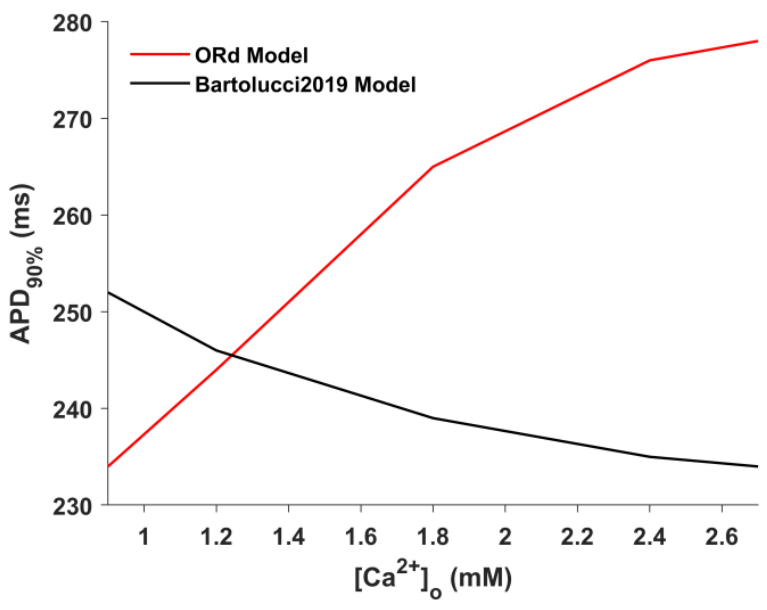

Figure 2. Comparison of the APD- $\left[\mathrm{Ca}^{2+}\right]_{\mathrm{o}}$ relationship for ORd and BS models in the range $0.9-2.4 \mathrm{mM}$.

While the ORd and BS models produced almost the same AP and $\mathrm{I}_{\mathrm{CaL}}$ (Figure 1, upper panels), $\left[\mathrm{Ca}^{2+}\right]_{\text {o }}$ changes produced opposite APD variations (Figure 2). The inverse relationship between APD and $\left[\mathrm{Ca}^{2+}\right]_{0}$ was indeed correctly reproduced by the new model. These simulations were obtained using $\left[\mathrm{K}^{+}\right]_{\mathrm{o}}=5.4 \mathrm{mM}$ since this is the commonly accepted physiological value for extracellular potassium.

The BS model well reproduced the same experimental data proposed in the original ORd model: APD rate dependence, APD restitution, effects of drug blocks, rate dependence of intracellular ion concentrations, APD rate adaptation (not shown).

\section{2 EADs}

We used the experimentally-calibrated population of in silico ventricular cells to study the occurrence of repolarization abnormalities in response to the rapid delayed rectifier potassium current block. Of these, 89 models developed early after-depolarizations and 88 models failed to repolarize (Figures 3 and 4). We also challenged our in silico population with high rate stimulation to assess alternans occurrence and rate adaptation, obtaining full adaptation in 897 models, alternans in 101 models and adaptation failure to increasing pacing rate in 308 models (not shown).

\section{Discussion and Conclusions}

The inverse APD- $\left[\mathrm{Ca}^{2+}\right]_{\mathrm{o}}$ dependence, missing in the Ord model, has been properly simulated by replacing the original $\mathrm{I}_{\mathrm{CaL}}$ with a Markov model, together with the other changes in $\mathrm{Ca}^{2+}$-handling.

APD rate dependence and restitution experimental data
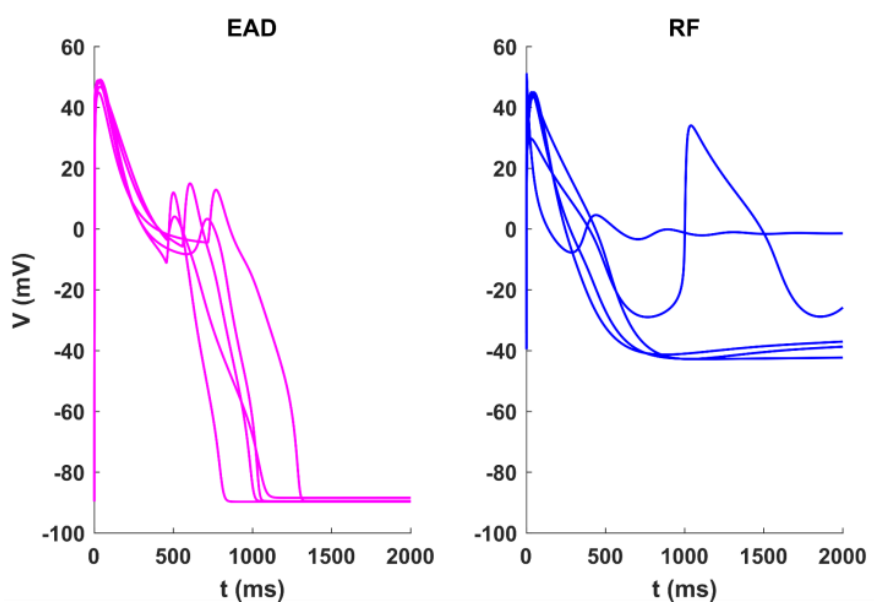

Figure 3. Examples of different response to dofetilide (0.1 $\mu \mathrm{M}, \quad \mathrm{CL}=4 \mathrm{~s} \quad[15])$ : models developing EADs (magenta, EAD, left panel) or failing to repolarize (blue, $\mathrm{RF}$, right panel).

(as well as other data (e.g. $\left[\mathrm{Na}^{+}\right] \mathrm{i}$ vs frequency relationship, etc.) are well reproduced by the BS model.

The population approach unveils different responses to drugs and potential arrhythmic events in control cells, as well as the occurrence of alternans.

In conclusion this study highlights the importance of reproducing, in simulation, conditions as close as possible to the ones used in the experiments to allow an effective comparison between experiments and simulations for validation.

It also offer to the computational scenario for a new updated human ventricular model. Indeed, The BS model expands the domain of applicability of the currently available computational models of human cardiac ventricular AP, suitable to explore changes in ventricular electrophysiology induced by electrolyte changes (e.g. effects of hemodialysis or pathological changes in $\mathrm{Ca}^{2+}$ concentrations) and the mechanisms of drug- or diseaseinduced pro-arrhythmic events.

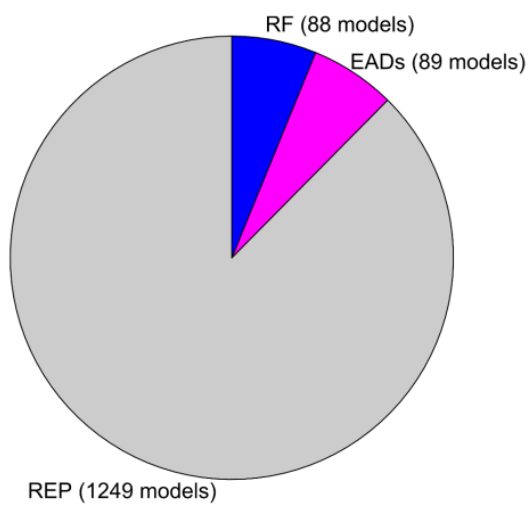

Figure 4. Models subdivision into REP, EADs and RF. 
A limitation of the model is that a clear distinction between identification and validation data was not possible due to limited availability of human data. Open issues are the choice of a single compartment for the SR and by the possibility of reproducing graded calcium release.

\section{References}

[1] O'Hara T, Virág L, Varró A, Rudy Y. Simulation of the undiseased human cardiac ventricular action potential: model formulation and experimental validation. PLoS Comput Biol 2011;7.

[2] Severi S, Corsi C, Cerbai E. From in vivo plasma composition to in vitro cardiac electrophysiology and in silico virtual heart: the extracellular calcium enigma. Philos Trans A Math Phys Eng Sci 2009;367:2203-23.

[3] Grandi E, Pasqualini FS, Pes C, Corsi C, Zaza A, Severi S. Theoretical investigation of action potential duration dependence on extracellular $\mathrm{Ca} 2+$ in human cardiomyocytes. J Mol Cell Cardiol 2009;46:332-42.

[4] Britton OJ, Bueno-Orovio A, Van Ammel K, Lu HR, Towart R, Gallacher DJ, et al. Experimentally calibrated population of models predicts and explains intersubject variability in cardiac cellular electrophysiology. Proc Natl Acad Sci 2013;110:E2098-105. doi:10.1073/pnas.1304382110.

[5] Muszkiewicz A, Britton OJ, Gemmell P, Passini E, Sánchez $\mathrm{C}$, Zhou $\mathrm{X}$, et al. Variability in cardiac electrophysiology: using experimentally-calibrated populations of models to move beyond the single virtual physiological human paradigm. Prog Biophys Mol Biol 2015;120:115-27. doi:10.1016/j.pbiomolbio.2015.12.002.

[6] Sager PT, Gintant G, Turner JR, Pettit S, Stockbridge N. Rechanneling the cardiac proarrhythmia safety paradigm: a meeting report from the cardiac safety research consortium. Am Heart J 2014;167:292-300.

[7] Colatsky T, Fermini B, Gintant G, Pierson JB, Sager P, Sekino Y, et al. The Comprehensive in Vitro Proarrhythmia Assay (CiPA) initiative - update on progress. J Pharmacol Toxicol Methods 2016;81:1520.

[8] Passini E, Severi S. Computational analysis of extracellular calcium effects on an improved human ventricular action potential model. Comput. Cardiol., 2013, p. 873-6.

[9] Bartolucci C, Passini E, Severi S. Optimization of the O'Hara-Rudy model of human ventricular action potential with respect to electrolyte concentrations and rate dependence. Comput Cardiol 2018;2018September:2-5. doi:10.22489/CinC.2018.333.

[10] Paci M, Pölönen R, Cori D, Penttinen K. Automatic optimization of an in silico model of human iPSC derived cardiomyocytes recapitulating calcium handling abnormalities. Front Physiol 2018;9:1-14.

[11] Li Z, Dutta S, Sheng J, Tran PN, Wu W, Colatsky T. A temperature-dependent in silico model of the human ether-à-go-go- related ( hERG ) gene channel. J Pharmacol Toxicol Methods 2016;81:233-9.

[12] M. D. McKay RJB and WJC. A comparison of three methods for selecting values of input variables in the analysis of output from a computer code. Technometrics 1979;21:239-45. doi:10.2307/1268522.

[13] Passini E, Britton OJ, Lu HR, Rohrbacher J, Hermans AN, Gallacher DJ, et al. Human in silico drug trials demonstrate higher accuracy than animal models in predicting clinical pro-arrhythmic cardiotoxicity. Front Physiol 2017;8:1-15. doi:10.3389/fphys.2017.00668.

[14] Dutta S, Mincholé A, Quinn TA, Rodriguez B. Electrophysiological properties of computational human ventricular cell action potential models under acute ischemic conditions. Prog Biophys Mol Biol 2017;129:40-52.

doi:10.1016/j.pbiomolbio.2017.02.007.

[15] Guo D, Liu Q, Liu T, Elliott G, Gingras M, Kowey PR, et al. Electrophysiological properties of HBI-3000: a new antiarrhythmic agent with multiple-channel blocking properties in human ventricular myocytes. J Cardiovasc Pharmacol 2011;57:79-85. doi:10.1097/FJC.0b013e3181ffe8b3.

[16] Shampine LF, Reichelt MW. The MATLAB ODE Suite. SIAM J Sci Comput 1997;18:1-22.

Address for correspondence:

Stefano Severi

Department of Electrical, Electronic

and Information Engineering,

University of Bologna,

Via dell'Università 50, 47522 Cesena $(\mathrm{FC})$, Italy

stefano.severi@unibo.it 